\title{
ROSTMEMA: A Double-Blind Randomized Placebo- Controlled Trial with the PDE4 Inhibitor Roflumilast in Patients Suffering from Long-term Cognitive Sequela After Stroke
}

Jill Kerckhoffs ( $\mathbf{j}$ j.kerckhoffs@maastrichtuniversity.nl)

Maastricht University Faculty of Psychology and Neuroscience: Universiteit Maastricht Faculty of Psychology and Neuroscience https://orcid.org/0000-0003-1815-0632

Arjan Blokland

Maastricht University Faculty of Psychology and Neuroscience: Universiteit Maastricht Faculty of Psychology and Neuroscience

Jos Prickaerts

Maastricht University Faculty of Health Medicine and Life Sciences: Universiteit Maastricht Faculty of Health Medicine and Life Sciences

leke Winkens

Maastricht University Faculty of Psychology and Neuroscience: Universiteit Maastricht Faculty of Psychology and Neuroscience

\section{Research Article}

Keywords: Cerebrovascular Disorders, Memory, Cognition, Functional Recovery, Drug Intervention, Plasticity, Phosphodiesterase inhibitor, Roflumilast

Posted Date: December 10th, 2021

DOl: https://doi.org/10.21203/rs.3.rs-968589/v1

License: (9) (1) This work is licensed under a Creative Commons Attribution 4.0 International License. Read Full License 


\section{Title}

ROSTMEMA: A double-blind randomized placebo-controlled trial with the PDE4 inhibitor roflumilast in patients suffering from long-term cognitive sequela after stroke

\section{Names protocol contributors}

Project leader

Principal investigator

Other investigator(s)
Dr. leke Winkens Department of Neuropsychology and Psychopharmacology, Faculty of Psychology and Neuroscience, Maastricht University. P.O. Box, 6200 MD Maastricht, The Netherlands tel: +31 (0)43 3884512 i.winkens@maastrichtuniversity.nl Dr. leke Winkens Department of Neuropsychology and Psychopharmacology, Faculty of Psychology and Neuroscience, Maastricht University. P.O. Box, 6200 MD Maastricht, The Netherlands tel: +31 (0)4338 84512 i.winkens@maastrichtuniversity.nl Prof. Dr. Arjan Blokland a.blokland@maastrichtuniversity.nl Prof. Dr. Jos Prickaerts jos.prickaerts@maastrichtuniversity.nl PhD student: Jill Kerckhoffs, Msc (j.kerckhoffs@maastrichtuniversity.nl

\section{Abstract}

- Background: The aim of this study is to examine whether treatment with roflumilast improves cognition in patients suffering from cognitive sequelae after stroke. The results may provide a proof of concept on the potential of roflumilast (or other phosphodiesterase-4 (PDE-4) inhibitors) as pharmacotherapeutic treatment to enhance cognition, and will further increase our knowledge on the role of PDE4 in human cognition in general. Cognitive processes, in particular memory, will be assessed.

- Methods: 100 female and male patients (41-70 years old) suffering from cognitive complaints 1 year after stroke will be recruited via advertisements via social media and via local caretaking organizations. The first phase of the study will be conducted according to a double-blind, randomized placebocontrolled, between-subjects design. After a baseline measurement, participants will be tested for acute (1 hour after drug intake) and subsequent chronic (1.5 and 3 months after start treatment) treatment effects. In a second phase, the placebo group (50 people) will be given the opportunity to receive roflumilast. This is an open label design. The roflumilast group will be tested for long-term treatment effects at three months after the end of treatment. 
- Discussion: Strengths of the current study are the open-label design as well as the assessment of longterm effects. The findings of the current study will demonstrate if the mechanism of PDE4 inhibition is a relevant target to improve cognitive functions in patients with brain damage after a stroke. If positive effects are found in this patient group, this treatment could also be relevant for other brain disorders (e.g. head trauma, mild cognitive impairment, Fragile $X$ syndrome, schizophrenia) in which enhanced neuronal plasticity is required to improve cognition.

- Trial registration: The Medical Ethics Committee of Maastricht University Medical Center+ granted ethics approval of the fourth version of the protocol on February 25, 2021. The trial was registered at the European Drug Regulatory Affairs Clinical Trials (EudraCT) register on July 29, 2020, https://www.clinicaltrialsregister.eu/ctr-search/search?query=2020-003768-16 and Clinicaltrials.gov (NCT04854811) at April 21, 2021, https://clinicaltrials.gov/ct2/show/NCT04854811. The Central Committee on Research Involving Human Subjects (CCMO) granted approval on May 4, 2021.

\section{Keywords}

Cerebrovascular Disorders

Memory

Cognition

Functional Recovery

Drug Intervention

Plasticity

Phosphodiesterase inhibitor

Roflumilast

\section{Administrative information}

Note: the numbers in curly brackets in this protocol refer to SPIRIT checklist item numbers. The order of the items has been modified to group similar items (see http://www.equator-network.org/reportingquidelines/spirit-2013-statement-defining-standard-protocol-items-for-clinical-trials/).

\begin{tabular}{|l|ll|}
\hline Title $\{1\}$ & $\begin{array}{l}\text { ROSTMEMA: A double-blind randomized placebo-controlled trial, with } \\
\text { the PDE4 inhibitor roflumilast in patients suffering from long-term } \\
\text { cognitive sequela after stroke }\end{array}$ \\
\hline Trial registration $\{2 \mathrm{a}$ and $2 \mathrm{~b}\}$. & $\begin{array}{l}\text { EudraCT number } \\
\text { Protocol ID }\end{array}$ & 2020-003768-16 \\
& Clinicaltrials.gov & NCT048587.068.20 \\
& & \\
\hline
\end{tabular}




\begin{tabular}{|c|c|c|}
\hline Protocol version $\{3\}$ & \multicolumn{2}{|c|}{$4^{\text {th }}$ draft, February 2021} \\
\hline Funding $\{4\}$ & Subsidizing party & $\begin{array}{l}\text { Dutch Brain } \\
\text { Foundation } \\
\text { (DR-2019-003) }\end{array}$ \\
\hline Author details $\{5 a\}$ & $\begin{array}{l}\text { Dr. leke Winkens } \\
\text { Department of Neuro } \\
\text { of Psychology and N } \\
\text { P.O. Box, } 6200 \text { MD N } \\
\text { tel: +31 (0)43 } 38845 \\
\text { Other investigators: } \\
\text { Prof. Dr. Arjan Blokla } \\
\text { Prof. Dr. Jos Prickaer } \\
\text { Jill Kerckhoffs, MSc ( }\end{array}$ & $\begin{array}{l}\text { hology and Psychopharmacology, Faculty } \\
\text { science, Maastricht University.. } \\
\text { tricht, The Netherlands } \\
\text { winkens@maastrichtuniversity.nl } \\
\text { a.blokland@maastrichtuniversity.nl) }{ }^{2} \\
\text { os.prickaerts@maastrichtuniversity.nl }{ }^{3} \\
\text { ckhoffs@maastrichtuniversity.nl) }\end{array}$ \\
\hline $\begin{array}{l}\text { Name and contact information for } \\
\text { the trial sponsor }\{5 b\}\end{array}$ & Sponsor & University of Maastricht (UM) \\
\hline Role of sponsor $\{5 c\}$ & $\begin{array}{l}\text { This study is subsidiz } \\
\text { 00310). The funder } \\
\text { process of the study } \\
\text { developed the study } \\
\text { the data, the manage } \\
\text { data and the decision }\end{array}$ & $\begin{array}{l}y \text { the Dutch Brain Foundation (DR2019- } \\
\text { ded marginal input during the review } \\
\text { gn. The sponsor (Maastricht University) } \\
\text { gn and will be responsible for collection of } \\
\text { t, the analysis and the interpretation of the } \\
\text { ubmit the report for publication. }\end{array}$ \\
\hline
\end{tabular}

\section{Introduction}

\section{Background and rationale $\{6 a\}$}

A stroke has various effects on brain functions and can have a wide variety of consequences, depending on location and severity of the ischemic event. In a consensus meeting, stroke patients, caregivers and health 
professionals agreed on 10 top priorities in stroke research (1). As a first priority they listed 'What are the best ways to improve cognition after stroke?'. Although the other nine impairments are also considered important, there is a good consensus that cognitive impairments are of great concern. In line with this, cognitive impairment is considered a major determinant of poor long-term disease outcome (2). Moreover, when asking patients about their major complaints they mention that the cognitive impairments lie at the core of many other problems they experience $(3,4)$. It is quite important to note that currently there are no drug treatments available for stroke patients who suffer from cognitive problems $(5,6)$. Current rehabilitation treatments mainly aim at learning to cope with the lasting impairments. Any gain in treating these cognitive functions would be a major benefit for the patient.

Stroke is associated with a damage of brain cells and poor functioning of the brain. Although the brain has the capacity to recover from the injury (connections and penumbra of ischemic tissue) the neurons will die in the core area of the ischemic tissue. Thus, therapies should aim at rescuing neurons in the penumbra region and restore brain functions on basis of neuroplasticity in those brain areas that take over functions of damaged brain areas. It is well known that the cyclic adenosine monophosphate (cAMP) plays an essential role in neuronal plasticity and that PDE4 inhibitors can increase the level of cAMP signaling (7). The positive effect of PDE4 inhibition on neuronal plasticity has been shown in various pre-clinical models. Of note, this mechanism of action is very different from that of other drugs (e.g., cholinesterase inhibitors, modafinil) that have been tested unsuccessfully in stroke patients thus far (5). The effects of roflumilast on neuronal plasticity could suggest that treatment could lead to long lasting changes in the brain and that people may have longterm benefits from roflumilast treatment. This will be investigated in the current study.

PDE4 inhibition has shown to improve cognitive and affective functions in different animal models of ischemic stroke $(8,9,10,11)$. These findings strongly support the notion that the underlying mechanism of action of PDE4 inhibition is relevant in reversing brain functions after stroke. Actually, three different mechanisms of action have been suggested for PDE4 inhibition: an anti-inflammatory effect, a neuroprotective effect, or an effect on cortical reorganization. Another more direct mechanism of action could be suggested based on a study that reported an upregulation of PDEs (including PDE4) after brain injury (12). An upregulation of PDEs leads to a higher degradation of CAMP that will have a negative effect on intracellular signaling and neuroplasticity (e.g. less dendrites and synapses) in neurons (i.e., long-term potentiation). Thus, a PDE inhibitor has a positive effect on intracellular signaling and neuroplasticity (e.g. more dendrites and synapses) by inhibiting the degradation of CAMP. This positive effect of PDE4 inhibition on plasticity has been shown in two different brain injury models and one study actually demonstrated the effect with roflumilast (12, 13).

An interesting finding is that PDE4 inhibition can have an effect on brain recovery when administered three months after inducing traumatic brain injury (14). First, this is one of the very few studies that shows that drug treatment can have an effect on brain function when given at an extended period after brain injury. Secondly, this study strongly supports the notion that PDE4 inhibition has mainly an effect on neuronal plasticity rather than a neuroprotective or anti-inflammatory effect. Of note, this would be a preferred action for our study in patients who are more than one year post stroke. PDE4 inhibitors have been reported to reduce depression-like behaviors in pre-clinical studies (15). If this could be translated to humans this would be a very 
favorable combination of effects of PDE4 inhibitors since it would treat two very important and often cooccurring symptoms in stroke patients.

PDE4 inhibitors have been investigated in healthy adults and elderly people $(16,17)$. Although PDE4 inhibitors have been suggested to have a great potential to treat brain disorders, the development of PDE4 inhibitors as therapeutic drugs has been hampered by their dose-limiting emetic side effects (e.g., nausea, dizziness, diarrhea). An example of such a classic PDE4 inhibitor is rolipram (Shering AG), which was developed as a possible antidepressant in the 1980s. The current PDE4 inhibitors that have been developed, show a strong reduction in emetic side effects. Roflumilast (AstraZeneca) is such an example. In 2010, roflumilast was approved as an anti-inflammatory drug under the name of Daxas (European Union, EU) for the treatment of chronic obstructive pulmonary disease (COPD) exacerbations (18). In previous studies it has been shown that roflumilast has very limited side effects and in fact does not differ from the reported side effects of participants who received placebo (19). Its far more favorable efficacy-side effect profile allowed our first promising studies in healthy adults and healthy elderly $(19,20,21)$. The positive effects of roflumilast on memory performance in different studies were found at a low dose $(19,20)$. This provides a favorable therapeutic window for this drug. Thus, considering this feature of roflumilast this offers the unique opportunity to investigate the promising clinical potential of a PDE4 inhibitor in a study with stroke patients. Recently, a study showed beneficial effects of chronic treatment with a novel PDE4 inhibitor on cognitive performance and daily functioning in Fragile $X$ patients (22)

Summarizing, previous studies confirm that PDE4 inhibitors have a favorable effect on neuroplasticity. Therefore, the next step will be to test whether PDE4 inhibitors (e.g. roflumilast) could be used to treat patients suffering from long-term cognitive sequela after stroke. The aim of this study is to examine whether roflumilast improves cognition in patients suffering from cognitive sequelae after stroke. The results may provide a proof of concept on the potential of roflumilast (or other PDE4 inhibitors) as pharmacotherapeutic treatment to enhance cognition, and will further increase our knowledge on the role of PDE4 in human cognition in general.

\section{Objectives $\{7\}$}

The primary objective of this study is to show that roflumilast can improve verbal memory performance in patients who suffered a stroke. The Rey's Verbal Learning Test (VLT) will be used and the delayed recall performance (after about $30 \mathrm{~min}$ ) will be measured. This test has been found to be sensitive for the positive effects of roflumilast in our previous studies. The test measures episodic memory that reflects memory for personal events.

Our secondary objective is aimed at measuring effects on other cognitive functions (reaction time, attention, executive functions), on aspects of every day memory, activities in daily living, and well-being. Hypotheses

The main hypotheses for this study have been formulated as follows:

1. Acute (1 hour) and chronic ( 1.5 and 3 months) treatment with $100 \mu \mathrm{g}$ roflumilast, compared to placebo, will improve episodic memory, in patients with long-term cognitive complaints after stroke;

2. Chronic treatment of 1.5 and 3 months with $100 \mu \mathrm{g}$ roflumilast, compared to placebo, will improve aspects of attention and executive functions and reaction time, everyday memory, and measures of general well-being 
(after 1.5- and 3 months of treatment), as assessed with different neuropsychological tests in patients with long-term cognitive complaints after stroke;

3. Roflumilast will lead to lasting improvements in cognitive performance and general well-being ( 3 months after roflumilast treatment stopped).

4. Roflumilast will lead to improved episodic memory, aspects of attention and executive functions and reaction time, everyday memory, and measures of general well-being (after 1.5-3 months of chronic treatment), in patients with long-term complaints after stroke. This will also be the case for the open-label design within subject-comparison, comparing previous placebo results with their known roflumilast treatment results.

\section{Trial design $\{8\}$}

The study will be conducted according to a double-blind, randomized placebo-controlled, between-subjects design. The study is a community based parallel group, two-arm ( $N=50$ per arm), superiority trial with 1:1 allocation ratio. Random assignment (randomized in blocks of 20 subjects) to placebo and $100 \mu \mathrm{g}$ roflumilast. The duration of treatment is planned for 3 months. The total duration of the study is 3.5 years, with the last inclusion of about 3 years into the study.

\section{Methods: Participants, interventions and outcomes}

\section{Study setting $\{9\}$}

Recruitment of participants will take place between May 2021 and September 2024. Patients who suffered from a stroke more than one year before inclusion will be recruited via local caretaking organizations and social media.

\section{Eligibility criteria $\{10\}$}

Patients who suffered a stroke at least one year ago are eligible for the trial if they comply with all of the following criteria:

- $\quad 41$ to 70 years of age;

- Suffered a stroke at least one year ago; and at the age of 40 or later

- Willingness to sign an IC;

- Body mass index (BMI) between 18.5 and 35;

- Objective memory complaint: memory performance on the delayed recall in the VLT below the normative score (corrected for education, sex and age) (24).

Exclusion criteria are:

- Normal Pressure Hydrocephalus (NPH)

- Morbus Huntington

- Parkinson's disease 
- HIVIAIDS

- Hepatitis C \& B

- Recent Transient Ischemic Attack (TIA) (< 1 years)

- Recent Cerebrovascular Accident (CVA) (<1 Years)

- People chronically treated for Asthma and COPD Gold Type 3 and 4

- History of schizophrenia, bipolar disorder or psychotic symptoms not otherwise specified or previous treatment for these disease (lifetime)

- Current affective disorder (i.e. anxiety or major depression) diagnosed by a clinician

- Cognitive problems due to alcohol abuse, brain tumor, epilepsy, encephalitis or lack of capacity to consent to participation

- Current treatment with (or illicit use of) cannabis, opiates, benzodiazepines, MDMA and cocaine

- Patients with moderate or major liver impairments will be excluded (e.g. Child-Pugh $B$ and $C$ ).

- Use of medication showing strong inhibition of either CYP3A4 or CYP1A2

- Patients with rare hereditary problems of galactose intolerance, Lapp lactase deficiency or glucosegalactose malabsorption

- Patients participating in other drug studies

\section{Who will take informed consent? $\{26 \mathrm{a}\}$}

People who are willing to participate will first visit Maastricht University for a medical screening. The researcher will obtain informed consent (IC) prior to the medical screening and baseline assessment, and after going over the study procedure.

\section{Additional consent provisions for collection and use of participant data and biological specimens $\{26 \mathrm{~b}\}$}

The participant signs an IC statement stating that they are well aware of the procedure(s) of the study, the data collection, and that they agree to participate in: a medical screening, filling in questionnaires, a neuropsychological examination. This includes that we may request information from their personal general practitioner (GP) and inform the GP about participation and possible important findings (e.g., during medical screening). On the consent form, participants will be asked if they agree to storage and use of their personal information for future research, as mentioned in the information letter and if they agree to be approached for participation in future studies. By signing the consent form, participants give permission to the use of their data, should they choose to withdraw from the study, for the research team to request injury-related information from their medical files and to share data with the regulatory authorities and the clinical research monitor of Maastricht University, where relevant. The participants are asked whether they would like to know in which treatment group they were after the end of the study. This trial does not involve collecting biological specimens for storage.

\section{Interventions}

\section{Explanation for the choice of comparators $\{6 \mathrm{~b}\}$}


Roflumilast is compared to placebo as there is no current pharmacological treatment for improving cognition one year after a stroke.

\section{Intervention description $\{11 \mathrm{a}\}$}

One dose of the investigational drug (Generic name: roflumilast, trade name: Daxas (EU), (Daliresp (US)) will be used. Treatments will be administered in identically appearing capsules to ensure double blinding. No other interventions are allowed during the test sessions. However, to stimulate the efficiency of roflumilast and increase the likelihood of improving neuronal plasticity, all participants (roflumilast and placebo group) perform computerized tests (Cogstate) (5 times a week, 15 minutes each time). There are no further restrictions or changes with regard to diet, other than prohibition from alcohol, illicit drugs (24 hours before testing), smoking and caffeinated drinks in the morning before test sessions.

\section{Dosages, dosage modifications and method of administration}

Participants will be instructed to take one capsule orally, every day at the same time, with water. The capsule can be taken with or without food. The capsules are kept in the pharmacy, or at the UM, department of Neuropsychology and Psychopharmacology, until they are distributed to the participant. The following dose levels will be used, as well as a placebo that only contains principal constituent lactose monohydrate.

- Roflumilast (Daxas $\left.{ }^{8}\right), 100 \mu \mathrm{g}$ orally

- Placebo, orally

In order to obtain the favored dosages and an identical appearing placebo, the tablets will be pulverized, and the appropriate quantities will be distributed over capsules (according to Good Manufacturing Practice (GMP)). Each roflumilast pill for oral administration contains the following inactive ingredients: lactose monohydrate, corn starch, povidone, and magnesium stearate. The placebo tablet will only contain the principal constituent lactose monohydrate. (For more information about roflumilast: https://www.accessdata.fda.gov/drugsatfda docs/nda/2011/0225220rig1s000ClinPharmR.pdf)

A systematic review of medication non-adherence in individuals with cognitive impairment has shown that post-stroke patients are at risk for non-adherence for various reasons (23). Accordingly, a so-called "roflumilast medication-diary" will be included to increase the medication adherence of the intervention. This diary and all the information will be discussed in the first test session at home. The diary will inform them on how best to store and take roflumilast, and what is advisable if they have forgotten to take a dose. On this diary, participants will be able to note daily whether they have taken roflumilast and at which time point. An additional space has been added to report eventual particularities, comments and more. Participants will receive extra capsules in case of accidents i.e. dropping a tablet in the sink, but these additional capsules are also included to examine adherence. Since we will count the remaining pills and see if this matches what they wrote down in their diary about intake. Lastly, we will make an effort to involve a committed informal caregiver or family relative of the participant for supporting the patient regarding the medication adherence.

\section{Preparation and labelling of Investigational Medicinal Product}


A GMP certified manufacturer has been appointed to take care of ordering and reprocessing of roflumilast tablets into capsules with our defined doses. Roflumilast tablets will be crushed to a powder and the powder will be mixed with filler lactose monohydrate in the appropriate proportions. Size 0 capsules will be manufactured with 0 and $100 \mu \mathrm{g}$ roflumilast.

\section{Drug accountability}

The roflumilast and placebo capsules will be produced by a GMP certified manufacturer. The manufacturer will receive a randomization list from an independent researcher (not involved in the project) and will label the jars. The manufacturer will ship the jars in 6 batches (about every half year). For each participant 2 jars each containing 50 pills (100 $\mathrm{\mu g}$ roflumilast or placebo) will be prepared on basis of the randomization list. The pharmacy (MUMC+) will take care of the storage of the jars with the capsules. Upon delivery, the complete batch will be controlled and an inventory form will be signed in case of positive evaluation of the batch. An independent researcher will send the pharmacy the randomization list beforehand. Researchers that are part of the study will be able to pick up the batches by means of a prescription that will be provided by the medical doctor involved in the study. The drugs will then be stored at the UM, department of Neuropsychology and Psychopharmacology until it is given to the participant. At the start of the study the participants will receive a jar of 50 pills for the first 1.5 months. After the second test day (1.5 months later), they will receive the second and last jar of 50 pills for the following 1.5 months. Accordingly, the participants and the researchers involved in the project will stay blinded.

\section{Criteria for discontinuing or modifying allocated interventions $\{11 \mathrm{~b}\}$}

Participants can leave the study at any time for any reason if they wish to do so, without any consequences and without providing an explanation on one's reason. Screening procedures at baseline might reveal anomalies of which the subject is unaware. In case of clinical relevance, the participant and their GP will be informed about the nature of the finding. Participants that do not agree with this procedure are not allowed to participate in the study. The investigator can decide to withdraw a participant from the study if he/she does not comply with the rules of the experiment. Furthermore, if the participant shows side effects that may interfere with the performance on the tasks, the investigator can, together with the participant, decide to either reschedule a test day or withdraw the participant from the experiment. In case the researcher suspects that the participant is not mentally competent to further decide whether or not to continue the study, it can be decided after deliberation with a medical doctor that the participant has to withdraw from the study.

\section{Strategies to improve adherence to interventions $\{11 \mathrm{c}\}$}

At every test session, the amount of capsules the participant has left will be checked by the researcher. Participants fill out a daily medication-diary to monitor intake and side effects. The researcher offers weekly phone calls to check up on the participants and remind them of taking their medication and filling out their diary. At the first assessment the participants are asked whether they would like the weekly phone calls or whether they would like to include their caregiver; the caregivers can be included in the process by accompanying the patient to the baseline session and helping the patient with a daily reminder. The researcher will check whether the participant completes the daily computer tasks. If not, the researcher will 
contact the participant.

\section{Relevant concomitant care permitted or prohibited during the trial $\{11 \mathrm{~d}\}$}

All participants are allowed to receive any form of care that they need. Participants are asked not to participate in any other drug trials or start treatment for their cognitive problems.

\section{Provisions for post-trial care $\{30\}$}

Medication will not be distributed after the trial. Long-term beneficial effects on cognition and daily activities could be expected by current treatment alone. The sponsor/investigator has a liability insurance, which is in accordance with article 7 of the WMO. The sponsor (also) has an insurance, which is in accordance with the legal requirements in the Netherlands (Article $7 \mathrm{WMO}$ ). This insurance provides cover for damage to research subjects through injury or death caused by the study. The insurance applies to the damage that becomes apparent during the study or within 4 years after the end of the study.

\section{Outcomes $\{12\}$}

\section{Main study parameter/endpoint}

The main study parameter is cognitive performance using a test battery in which verbal memory is the primary outcome measure as assessed with the VLT (Rey's Verbal Learning Test; 15 words) (24). Outcome scores are defined as number of recalled items in the immediate (sum of 5 trials) and the delayed test. This includes five times immediate recall, and a $30 \mathrm{~min}$ delayed recall. The changes from baseline to acute effects, as well as six weeks and three months into treatment and long-term effects will be statistically analyzed.

\section{Secondary study parameters/endpoints}

The changes from baseline to acute effects, as well as six weeks and three months into treatment and longterm effects will be analyzed for all secondary study parameters.

For measuring effects on cognitive functions other than memory, the Letter-Digit Substitution Test (LDST), Trail Making Test (TMT) and a reaction time test (simple reaction time, movement time) will be used.

The LDST is a paper-and-pencil cognitive test presented on a single sheet of paper that requires a subject to match symbols to numbers according to a key located on the top of the page (25). The number of correct symbols within the allowed time, constitutes the score. The test has high discriminate validity, and is sensitive to change in cognitive function across a wide range of clinical populations.

The TMT is a neuropsychological test of visual attention and mental flexibility (26). The test is widely used to assess executive functioning in patients with stroke.

The Reaction Time Task is a computer task in which participants have to react to stimuli by pressing a button. The dependent variables of this task consists of median initiation times (time needed to release the button) and median movement times (time needed to move from one button to the target button) of correct choices. 


\section{The more general effects of roflumilast treatment}

The changes from baseline to acute effects, as well as six weeks and three months into treatment and longterm effects will be analyzed for all secondary study parameters.

Other important clinical tests will be used to measure the more general effects of roflumilast treatment on daily memory and activities of daily living. These will inform us about more clinically relevant effects. For this, the Everyday Memory Questionnaire-Revised (EMQ-R), the Rivermead Behavioural Memory Test (RBMT), and a test for daily participation (Utrecht Scale for Evaluation of RehabilitationParticipation (USER-Participation)) will be used.

The EMQ-R (27) is a questionnaire which requires participants to respond to a list of memory-related behaviors by providing an estimate of how many times this happened to them over the previous month. Higher scores are indicative of greater presence of memory difficulties. It is a valid and reliable tool that has good face validity for use with neurological patients.

The RBMT is a functional memory assessment evaluating an individual's ability to use memory function for the performance of daily tasks, and attempts to bridge conventional and behavioral procedures (28). The RBMT subtest 'stories' will be used to assess a person's ability to absorb verbal information. The RBMT has demonstrated a very high inter-rater reliability and good alternate form reliability (correlation between performances on parallel forms). This allows for repeated assessments.

The USER-P is a generic measure of participation (29). It is divided into three scales: Frequency, Restrictions, and Satisfaction. The sum of scores for the scales are based on the items that are applicable to the patient's situation and each sum score is converted to a 0-100 scale with higher scores indicating better participation (i.e., higher frequency, less restrictions, higher satisfaction). The USER-P has adequate reliability and validity in patients with physical disabilities.

\section{Mood wellbeing and side-effects}

The Hospital Anxiety and Depression Scale (HADS) is used to determine levels of anxiety and depression that the patient is experiencing (30). Bjelland et al. (31) have concluded through a large review of studies, that the identified cut-off point is 8 out of 21 for an anxiety or depression.

\section{Complaints and side-effects}

In order to monitor whether the participants are experiencing any complaints or side-effects, simply whether they have noticed any side-effects since starting roflumilast will be inquired. These side-effects and complaints will be noted down in the medication diary. The phone-monitoring schedule will be once every week the first month and the following months, every two weeks.

\section{Participant timeline $\{13\}$}

The schedule of screening, randomization, efficacy, safety, study drug and other medication can be found in Table 1. All participants will start with a medical screening and a baseline session, then approximately one week later they start with the first pill, an hour after first intake the acute session will be performed, then a session six weeks after the start of the treatment and three months after the start of the treatment, this will be 
the end of taking the medication. The roflumilast group will be asked for a long-term assessment three months after the end of the treatment. The placebo group will be asked whether they want to participate in the open label study, if so, they receive another three assessments.

Table 1. Study procedure

\begin{tabular}{|c|c|c|c|c|c|c|}
\hline Item & $\begin{array}{l}\text { Screen } \\
\text { Visit } 1\end{array}$ & $\begin{array}{c}\text { Baseline } \\
\text { (Test } \\
\text { session 1) } \\
\text { Visit 2 } \\
\end{array}$ & $\begin{array}{c}\text { Acute } \\
\text { (Test } \\
\text { session 2) } \\
\text { Visit 3 } \\
\end{array}$ & \begin{tabular}{|c|}
1.5 Months \\
(Test \\
session 3) \\
Visit 4 \\
\end{tabular} & $\begin{array}{c}\text { Months } \\
\text { (Test } \\
\text { session 4) } \\
\text { Visit } 5 \\
\end{array}$ & $\begin{array}{l}6 \text { Months } \\
\text { (Test } \\
\text { session 5) } \\
\text { Visit } 6 \\
\end{array}$ \\
\hline \multicolumn{7}{|l|}{ SCREENING } \\
\hline Informed consent & $\mathrm{x}$ & & & & & \\
\hline $\begin{array}{l}\text { Demography (date of birth, } \\
\text { sex, ethnic group, height) }\end{array}$ & $\mathrm{X}$ & & & & & \\
\hline $\begin{array}{l}\text { Psychosocial information } \\
\text { (Highest } \\
\text { education/occupation level } \\
\text { attained, present employ- } \\
\text { ment and living } \\
\text { arrangement) }\end{array}$ & $\mathrm{X}$ & & & & & \\
\hline $\begin{array}{l}\text { Medical /psychiatric history } \\
\text { /surgical history }\end{array}$ & $\mathrm{x}$ & & & & & \\
\hline Blood alcohol content & & $x$ & $x$ & $x$ & $x$ & $x$ \\
\hline $\begin{array}{l}\text { Final check of inclusion / } \\
\text { exclusion criteria }\end{array}$ & & $\mathrm{x}$ & & & & \\
\hline RANDOMIZATION & & & $\mathrm{X}$ & & & \\
\hline \multicolumn{7}{|l|}{ EFFICACY } \\
\hline Neuropsychological tests & & $\mathrm{x}$ & $\mathrm{x}$ & $\mathrm{X}$ & $\mathrm{x}$ & $\mathrm{X}$ \\
\hline Well-being test & & $\mathrm{x}$ & $x$ & $x$ & $x$ & $\mathrm{X}$ \\
\hline \multicolumn{7}{|l|}{ SAFETY } \\
\hline Adverse events & & & $\mathrm{X}$ & $x$ & $\mathrm{x}$ & $\mathrm{X}$ \\
\hline Physical examination & $\mathrm{X}$ & & & & & \\
\hline Vena puncture & $x$ & & & & & \\
\hline Heart rate/blood pressure & $x$ & $x$ & $x$ & $x$ & $x$ & $\mathrm{x}$ \\
\hline ECG & $x$ & & & & & \\
\hline \multicolumn{7}{|c|}{ STUDY DRUG AND OTHER MEDICATIONS } \\
\hline $\begin{array}{l}\text { Drug intake (roflumilast or } \\
\text { placebo) }\end{array}$ & & & $x$ & $x$ & $x$ & \\
\hline $\begin{array}{l}\text { Previous/concomitant } \\
\text { medication }\end{array}$ & $\mathrm{X}$ & $\mathrm{x}$ & $\mathrm{X}$ & $\mathrm{X}$ & $\mathrm{X}$ & $\mathrm{X}$ \\
\hline $\begin{array}{l}\text { Study medication } \\
\text { administration record }\end{array}$ & & & $\mathrm{x}$ & $\mathrm{X}$ & $\mathrm{x}$ & \\
\hline Drug return & & & & $\mathrm{x}$ & $\mathrm{x}$ & $\mathrm{X}$ \\
\hline
\end{tabular}


Note: The participants who received the placebo in the first leg of the study and are offered to take roflumilast for another three months. They will follow the same procedures as in the first leg of the study (but now open label).

\section{Sample size $\{14\}$}

The sample size calculation is based on a previous study in which a positive effect was found of roflumilast on memory performance in the verbal learning memory task that will also be used in the current study (Blokland et al. (20); differences between means $=2$, stdev $=3.35$ combined). In the previous study an effect size (Cohen's d) of 0.7 was obtained. This is a relative high effect size but this is based on our previous study and we would like to show a clinically relevant effect size. A power of 0.95 and test at $p<0.05$ will be used. Based on these parameters 45 patients per treatment group (roflumilast - placebo) are needed in our study (using G-power). A drop out of $10 \%$ (as experienced in the previous roflumilast studies) is anticipated, so 50 participants for each group will be included.

\section{Recruitment $\{15\}$}

Adverts will be used in order to inform people (starting in the Limburg region) about this research. The adverts will be spread via local caretaking organizations and via social media. People who are interested in the study can contact the researcher by phone or by mail, whose information is given in the advertisement. The researcher will give oral information about the study and send elaborate written information. After having received the written information, possible participants have seven days to consider their decision regarding the study participation. After seven days the researcher will contact the possible participant to answer remaining questions. In case of interest the researcher will make an appointment for the medical screening and baseline. At this meeting the participant and researcher will sign informed consent. Note that participants will be informed that they can retract and quit their participation at any point of the study. This will be included in the written information and the consent sheet.

\section{Assignment of interventions: allocation}

\section{Sequence generation $\{16 \mathrm{a}\}$}

Randomization and treatment allocation will be performed by an independent researcher, as the study will be conducted in a randomized double-blind manner. The allocation sequence will be generated by an independent researcher using a computerized random number generator (https://commentpicker.com/random-number-generator.php). The randomization will be a block randomization of 20 participants (randomization within these blocks). The randomization list will be send to the GMP certified manufacturer and the Hospital pharmacy.

\section{Concealment mechanism $\{16 b\}$}

The independent researcher sent the randomization list to the manufacturer. The GMP certified manufacturer will code the treatments and provide coded jars with the roflumilast pills. Roflumilast will be produced and shipped to the Hospital Pharmacy who will then deliver the medication to the researchers on 
basis of a prescription of the medical doctor.

\section{Implementation $\{16 c\}$}

The randomization list will be send to the GMP certified manufacturer and the clinical trial pharmacy. The researcher will enrol participants. Participants will be randomly assigned to the interventions by their participant number.

\section{Assignment of interventions: Blinding}

\section{Who will be blinded $\{17$ a}

The research is double-blind. Both the researchers as the participants are blinded.

\section{Procedure for unblinding if needed $\{17 \mathrm{~b}\}$}

Whenever unblinding would be necessary because of, for example, due to serious adverse events, the independent researcher or medical doctor will be asked to contact the medical doctor/General Practitioner of the subject. The researchers will be kept blind.

\section{Data collection and management}

\section{Plans for assessment and collection of outcomes $\{18 \mathrm{a}\}$}

Several methods will be used to obtain information on the outcomes. There will be several times of assessment. A baseline assessment, directly after the medical screening, an acute assessment at least one week after the baseline assessment, an assessment six weeks after the acute session, and an assessment three months after the acute session. After this the roflumilast group will be asked for another long term assessment three months after the end of treatment (six months after acute). The placebo group will be asked whether they would like to join for the open-label study and do another acute, six weeks and three months assessment. The variables measured in these assessments are described above (primary and secondary outcome measures). These outcome measures have been validated and all have a high reliability and validity. To make sure all outcomes measures are correctly gathered, all researchers performing the neuropsychological assessments are trained to administer the tests and questionnaires in the same and correct manner. All outcome measures are collected on paper, except for the Reaction Time Test, these are collected in computer files (Eprime). The data collected will eventually be stored in electronic data files (Excel, SPSS).

Next to this information from the medication-diary will be obtained, which will be filled out from the start of treatment till the end. This will be qualitative data. Other data that will be obtained are from the computer tasks which the participants will perform five times a week. 


\section{Plans to promote participant retention and complete follow-up $\{18 \mathrm{~b}\}$}

All participants will receive a voucher of $€ 20$ after each test session at home. There are at least four (to six) test sessions (depending on whether someone is in the placebo or medication group), which adds up to a total of $€ 80$ to $€ 120$ the end of the study. In the event of early termination of participation, payment will be made on the basis of the number of test days in which participation has taken place. Any expenses related to travelling costs will be reimbursed separately.

\section{Data management $\{19\}$}

This study will generate new data. Namely, a combination of quantitative and qualitative data. Accordingly, there will be no usage of reuse or combination of existing data. Data collection, entry and management will only take place at Maastricht University. Data will not leave Maastricht University. Intellectual property has already been established in a patent by the university.

The total amount of participants will be $\mathrm{N}=100$, which will result in a data collection of approximately 10 Gbytes. The study and its investigators will make use of the institution's standard facilities for storage and backup of the data, meaning that the data will be saved on a protected server and a daily back-up of this data is included. All data (i.e., IC forms, test material) will be stored for twenty-five years at the department of Neuropsychology and Psychopharmacology at Maastricht University. The data of the project will be discernible through dataverse (https://dataverse.nl/dataverse/maastricht). Handling of personal data will comply with EU General Data Protection Regulation and the Dutch Act on Implementation of the General Data Protection Regulation.

Data will be collected on paper and by computer. The raw data will be stored on paper and electronically in the data management files. The raw computer data will be stored and electronically saved in the data files. Data will be entered electronically twice by members of the research team any discrepancies between the two entries will be resolved by the person responsible for the second entry, or by discussion with a third researcher if necessary. The data collected will eventually be stored in electronic data files (Excel, SPSS). Manual range checks will be performed for demographic information. A logbook will provide with information on all activities in the electronic database. Access to electronic data is controlled by a password system, and access to original data will be restricted by storing the data in a locked cabinet (see 'Confidentiality).

\section{Confidentiality $\{27\}$}

All personal data (i.e., name, date of birth) from participants will be coded and will be handled confidentially, this data will be stored separately from the other research data. Each participant will receive a unique identification code, in ascending order, based on order of inclusion, starting at 2001 until 2100 . In order to link the data collected in the study to the patients, an identification code list will be made. The key to the code will be kept confidential by the principal investigators (i.e., Dr. I. Winkens and J. Kerckhoffs) of the study. Some people are allowed to see the medical and personal data. These include the principal investigators (i.e., Dr. I. Winkens and J. Kerckhoffs), Inspectie Gezondheidszorg en Jeugd (IGJ), and study monitors. The participant's data will not be disclosed to unauthorized third parties, and participant confidentiality will be maintained at all 
times. With permission of the participant, data will be stored for twenty-five years for use in future medical research on the further development of the use of roflumilast after stroke.

\section{Plans for collection, laboratory evaluation and storage of biological specimens for genetic or molecular analysis in this trial/future use $\{33\}$}

Blood and urines samples are collected only once for the medical screening procedure, they are analyzed by an external lab and will be destroyed directly after analyzing the results of the medical screening.

\section{Statistical methods}

\section{Statistical methods for primary and secondary outcomes $\{20 \mathrm{a}\}$}

Descriptive statistics and raw data handling

Descriptive statistics for continuous parameters will include sample sizes, mean and standard error of the dependent variable and covariates for each distinct level combination of factors. For categorical data, summary tables will present counts and percentages. Statistical summaries will be presented by treatment, sequence or period. Data will be checked for outliers and normality. Outliers (based on SPSS 'explore' test) will be deleted and in case of non-normality the data will be transformed in order to meet the normality criteria in order to apply parametric analysis. The transformation that will be applied depends on the data distribution.

\section{Difference scores and Mixed Models - Repeated Measures Design}

In order to estimate the improvement on the primary outcome measure the individual difference score will be calculated by subtracting the performance at baseline from the performance after 3 months of treatment. In addition, it will be analyzed whether the treatment has long-lasting effects by comparing the performance after 3 months of treatment with the performance 3 months later (when the participants did not receive treatment). We will correct for multiple comparisons (Bonferroni).

The repeated measures, which will be used for all primary and secondary outcome variables, will be analyzed using mixed models. The between-subject factor will be the treatment group (two levels: placebo and $100 \mu \mathrm{g}$ roflumilast). The response types within a task (type) will be entered as the within-subject factor, as well as time (T1 i.e. acute, T2 i.e. 1.5 months, T3 i.e. 3 months, T4 i.e. 3 months follow-up). These analyses will reveal whether the scores increase or decrease over time, and whether the effects are gradual (linear effect) or a more sudden effect (quadratic effect). An additional interaction term between treatment group and time is included to determine whether the treatment groups will lead to different trajectories over time. In addition, a regression model will be used in order to test the effects of age, sex and education level on the primary and secondary outcome measures. These analyses will give additional information as to which factors may underlie the main effect, and which factors do not contribute to the main effect. The significance level will be set at $\alpha=0.05$. 
For the open-label part, similar analyses will be done on all primary and secondary outcome variables, in order to:

1. Explore whether the effect is similar when patients know they receive roflumilast (here the results from the original roflumilast-group to the results of the open-label roflumilast group will be compared);

2. Have an extra control-analysis, by comparing the results of the original Placebo-group to the results of the open-label roflumilast-group (here each person serves as it own control - in this analysis treatment will be a within-subject factor).

\section{Interim analyses $\{21 b\}$}

No interim analyses are planned because there are no anticipated risks to participation in this study.

\section{Methods for additional analyses (e.g. subgroup analyses) $\{20 \mathrm{~b}\}$}

N.A.

\section{Methods in analysis to handle protocol non-adherence and any statistical methods to handle missing data $\{20 \mathrm{c}\}$}

If possible (enough power) analyses will be repeated with intention to treat analysis. The default approach to missing data is listwise deletion. This is a major advantage compared to repeated measures ANOVA. Listwise deletion is performed to remove the row of values when an observation is missing (imbalanced data result). Maximum likelihood is then used to get estimates of the parameters of the model. It is important to note that when data points are missing at random (MAR) or missing completely at random (MCAR), these estimates will be unbiased. If the data is not MAR it is expected to expect biased estimates.

\section{Plans to give access to the full protocol, participant level-data and statistical code $\{31 c\}$}

The full protocol, anonymized data set and statistical code will be available on request after the results of the study have been published. The data of the project will be discernible through dataverse (https://dataverse.nl/dataverse/maastricht).

\section{Oversight and monitoring}

Composition of the coordinating centre and trial steering committee $\{5 \mathrm{~d}\}$ 
Principal (IW, AB, JP), coordinating investigators (JK)

- Design of the study (IW, AB, JP);

- Preparation of protocol and revisions (IW, $A B$ );

- Ethics committee application (IW);

- Study planning (IW, JK);

- Recruiting, training and supervising research assistants (IW, JK);

- Responsible for trial master file (IW, JK);

- Provide annual reports to ethics committee (IW);

- Data verification (IW, JK);

- Publication of study reports (IW, AB, JP, JK);

Composition of the data monitoring committee, its role and reporting structure $\{21 \mathrm{a}\}$

Because of the low burden and minimal risks, no data monitoring committee was appointed.

\section{Adverse event reporting and harms $\{22\}$}

Adverse events (AEs)

Adverse events are defined as any undesirable experience occurring to a subject during the study, whether or not considered related to roflumilast. All AEs reported spontaneously by the subject or observed by the investigator or his/her staff will be recorded.

\section{Serious adverse events (SAEs)}

A serious adverse event is any untoward medical occurrence or effect that

- results in death;

- is life threatening (at the time of the event);

- requires hospitalization or prolongation of existing inpatients' hospitalization;

- results in persistent or significant disability or incapacity; 
- is a congenital anomaly or birth defect; or

- any other important medical event that did not result in any of the outcomes listed above due to medical or surgical intervention but could have been based upon appropriate judgement by the investigator.

- An elective hospital admission will not be considered as a serious adverse event.

All SAEs will be reported by the investigator through the web portal ToetsingOnline to the accredited MREC that approved the protocol, within 15 days after the research team has first knowledge of the serious adverse reactions. SAEs that result in death or are life threatening should be reported expedited. The expedited reporting will occur not later than seven days after the responsible investigator has first knowledge of the adverse reaction. This is for a preliminary report with another eight days for completion of the report.

\section{Suspected unexpected serious adverse reactions (SUSARs)}

Adverse reactions are all untoward and unintended responses to an investigational product related to any dose administered.

Unexpected adverse reactions are SUSARs if the following three conditions are met:

1. the event must be serious;

2. there must be a certain degree of probability that the event is a harmful and an undesirable reaction to the medicinal product under investigation, regardless of the administered dose;

3. the adverse reaction must be unexpected, that is to say, the nature and severity of the adverse reaction are not in agreement with the product information as recorded in:

- Summary of Product Characteristics (SPC) for an authorized medicinal product;

- Investigator's Brochure for an unauthorized medicinal product.

The sponsor will report expedited the following SUSARs through the web portal ToetsingOnline to the METC: - SUSARs that have arisen in the clinical trial that was assessed by the METC;

- SUSARs that have arisen in other clinical trials of the same sponsor and with the same medicinal product, and that could have consequences for the safety of the subjects involved in the clinical trial that was assessed by the METC.

The remaining SUSARs are recorded in an overview list (line-listing) that will be submitted once every half year to the METC. This line-listing provides an overview of all SUSARs from the study medicine, accompanied by a brief report highlighting the main points of concern.

The expedited reporting of SUSARs through the web portal Eudravigilance or ToetsingOnline is sufficient as notification to the competent authority.

The sponsor will report expedited all SUSARs to the competent authorities in other Member States, according to the requirements of the Member States.

The expedited reporting will occur not later than 15 days after the sponsor has first knowledge of the adverse reactions. For fatal or life threatening cases the term will be maximal seven days for a preliminary report with another eight days for completion of the report. 
Available data will be analyzed. In an unforeseen event, it could also be the case that the study has to be terminated. For example, if new data become available that there are potential issues with roflumilast treatment.

\section{Frequency and plans for auditing trial conduct $\{23\}$}

As this study falls under the scope of the Dutch Medical Research Involving Human Subjects Act (Dutch: wet medisch-wetenschappelijk onderzoek met mensen, WMO), the Clinical Trial Centre Maastricht (Maastricht University) appointed an independent clinical research monitor to the study. This person monitors whether the study is conducted according to the ICH-GCP guidelines and legislation and regulations. Together with the research team, a plan for monitoring was prepared, in which all details regarding the procedures are stated. This plan was made according to the specific demands of the study and in line with legislation and regulations. CTCM will give advice regarding laws and regulation, as well as control compliance of laws. CTCM will also carry out a regulation general control of the data collection, verification of source documents and case report forms (CRFs), inspect ICs and protocols, controlling the Trial Master File, and verify the reports on AEs and complications.

\section{Plans for communicating important protocol amendments to relevant parties (e.g. trial participants, ethical committees) $\{25\}$}

Amendments are changes made to the research after a favorable opinion by the accredited MREC has been given. All amendments will be notified to the MREC that gave a favorable opinion. A 'substantial amendment' is defined as an amendment to the terms of the MREC application, or to the protocol or any other supporting documentation, that is likely to affect to a significant degree:

- the safety or physical or mental integrity of the subjects of the trial;

- the scientific value of the trial;

- the conduct or management of the trial; or

- the quality or safety of any intervention used in the trial.

All amendments will be notified to the MREC, and to the competent authority.

\section{Dissemination plans $\{31 \mathrm{a}\}$}

All results from this research will be disclosed unreservedly in a scientific paper aimed for publication in a peer reviewed scientific journal. Besides this, all results will be presented to the CVA patient groups and practitioners and information will be shared and discussed in a user committee. The list of authors will include all individuals who made substantial contributions to conception and design, or analysis and interpretation of data, as well as drafting the paper or revising it for important intellectual content. Any publication will follow the CCMO guidelines (c.f., CCMO-statement Publication Policy). 


\section{Discussion}

A stroke has various effects on brain functions and can have a wide variety of lasting consequences for cognitive functioning. Currently there are no drug treatments available for stroke patients who one year after stroke still suffer from cognitive problems and no therapies are available that will actually improve the cognitive performance of CVA patients. Existing rehabilitation treatments mainly aim at improving physical functioning and learning to cope with the lasting cognitive impairments. Since cognitive impairments are considered to be at the core of many neuropsychological problems the patients are suffering from, any treatment that improves cognitive functions would lead to a better societal participation of these patients. The ultimate goal is to improve the quality of life. Since many of these patients will still live for about 30 years, an effective treatment would be very meaningful.

The aim of this study is to examine whether treatment with roflumilast improves cognition in patients suffering from cognitive sequelae after stroke. 100 female and male patients (41-70 years old) suffering from cognitive complaints at least one year after stroke will be recruited. The first phase of the study will be conducted according to a double-blind, randomized placebo-controlled, between-subjects design. The duration of treatment is planned for 3 months. After a baseline measurement, participants will be tested for acute ( 1 hour after drug intake) and subsequent chronic (1.5 and 3 months after start treatment) treatment effects. Possible lasting effects of roflumilast will be tested 3 months after treatment has ended. In a second phase, the placebo group (50 people) will be given the opportunity to receive roflumilast. This is an open label design. These participants also will be tested for acute ( 1 hour after drug intake) and chronic (1.5 and 3 months after start treatment) treatment effects.

The findings of the current study will demonstrate if the mechanism of PDE4 inhibition is a relevant target to improve cognitive functions in patients with brain damage after a stroke. The main hypotheses for this study are that treatment with $100 \mu \mathrm{g}$ roflumilast will lead to lasting improvements in episodic memory, aspects of attention and executive functions and reaction time, everyday memory, and measures of general well-being.

Strengths of the current study are the open-label design as well as the assessment of long-term effects. With the open-label study, it can be examined how patients perform when they know they are taking roflumilast, and this can be compared with the effects observed in the double-blind condition. This would be of great clinical significance: after all, in clinical practice, patients will know they are taking roflumilast. By measuring the long-term effects it can be measured whether the effects of roflumilast continue after treatment with roflumilast has stopped. These are valuable conclusions for future treatment options. For patients and doctors it would be interesting to know whether long-term treatment with pills is required or whether a temporary short-term treatment would be sufficient.

The possible risk factors in this study are the following. The actual number of patients recruited could be lower than expected. This could be prevented by using additional channels for recruitment or by increasing the region of recruitment. The participant's compliance with taking roflumilast could be lower than expected. However, the risk factor for this is considered low. Partners will be actively engaged in reminding the participants to take their medication. Another risk factor is that participants could drop out of the study because of possible negative side effects of roflumilast. From previous studies with higher doses of roflumilast we know that patients experience few negative side effects. For the current study the 
administered dose will be five times lower. Therefore we expect that patients will experience no to minimal side effects. The risk level for dropping out of the study due to negative side effects is rated as low. Clearly, lack of treatment effect is the most relevant risk factor for withdrawal from the study. Based on our previous studies in humans, this risk factor is also considered relatively low. Our previous studies in humans showed a positive effect of roflumilast on the outcome measures; these same outcome measures for memory performance are used in the current study.

The ultimate goal is to show that medical treatment with a PDE4 inhibitor can be used to improve cognitive functions of different patient populations and improve their quality of life. If positive effects can be found in this patient group, this treatment could also be relevant for other brain diseases (e.g. head trauma, mild cognitive impairment, Fragile $X$ syndrome, schizophrenia) in which enhanced neuronal plasticity is required to improve cognition.

\section{Trial status}

The Medical Ethics Committee of Maastricht University Medical Center+ granted ethics approval of the fourth version of the protocol on February 25, 2021. The trial was registered at the European Drug Regulatory Affairs Clinical Trials (EudraCT) register on July 29, 2020 and Clinicaltrials.gov (NCT04854811) at April 21, 2021. The CCMO granted approval on May 4, 2021.

Recruitment started in May, 2021. The first person was enrolled on July 20, 2021. Inclusion is currently ongoing and is expected to be completed in September, 2023.

\section{Abbreviations}

\section{AE}

AR

BMI

CAMP

CGMP

COPD

CRF

CTCM

CVA

CYP

LDST

EU

EudraCT

GCP

GMP
Adverse Event

Adverse Reaction

Body mass index

Cyclic Adenosine Monophosphate

Cyclic Guanosine Monophosphate

Chronic obstructive pulmonary

disease

Case Report Form

Clinical Trial Center Maastricht

Cerebrovascular accident

Cytochromes P450

Letter Digit Substitution Test

European Union

European Drug Regulatory Affairs

Clinical Trials

Good Clinical Practice

Good Manufacturing Practice 


$\begin{array}{ll}\text { GP } & \text { General Practitioner } \\ \text { HADS } & \text { Hospital Anxiety and Depression } \\ \text { IC } & \text { Scale } \\ \text { MAR } & \text { Missing at Random } \\ \text { MCAR } & \text { Missing Completely at Random } \\ \text { MREC } & \text { Medical Research Ethics } \\ & \text { Committee } \\ \text { MUMC } & \text { Maastricht University Medical } \\ \text { PDE } & \text { Center } \\ \text { RT } & \text { Phosphodiesterase } \\ \text { (S)AE } & \text { Reaction Time } \\ \text { (S)AR } & \text { (Serious) Adverse Event } \\ \text { SD } & \text { (Serious) Adverse Reaction } \\ \text { SUSAR } & \text { Standard Deviation } \\ & \text { Suspected Unexpected Serious } \\ \text { TIA } & \text { Adverse Reaction } \\ \text { UM } & \text { Transient Ischemic Attack } \\ \text { VLT } & \text { University of Maastricht } \\ \text { WMO } & \text { Rey's Verbal Learning Test } \\ & \text { Medical Research Involving } \\ & \text { Human Subjects Act; in Dutch: Wet } \\ & \text { Medisch-wetenschappelijk } \\ & \text { Onderzoek met Mensen } \\ & \end{array}$

\section{Declarations}

\section{Acknowledgements}

Not applicable.

\section{Authors' contributions $\{31 \mathrm{~b}\}$}

$A B, J P$ and IW developed the study protocol. AB, JP and IW contributed to the study design and procedures. $\mathrm{JK}$ wrote the first draft of this manuscript. All authors have read and approved the final version of this manuscript. 


\section{Funding $\{4\}$}

This study is supported by a grant from the Dutch Brain Foundation. The funder provided marginal input during the review process of the study design. The sponsor (Maastricht University) developed the study design and will be responsible for collection of the data, the management, the analysis and the interpretation of the data and the decision to submit the report for publication.

\section{Availability of data and materials $\{29\}$}

The datasets used and/or analyzed during the current study are available from the corresponding author on reasonable request after publication of the results.

\section{Ethics approval and consent to participate $\{24\}$}

The Medical Ethics Committee of Maastricht University Medical Center+ approved the trial (registration number METC20-068). The study will be conducted according to the principles of the Declaration of Helsinki (World Medical Association, October 2013) and in accordance with the Dutch Medical Research Involving Human Subjects Act (Dutch: WMO). All participants will provide informed consent prior to participation.

\section{Consent for publication $\{32\}$}

Not applicable

\section{Competing interests $\{28\}$}

$A B$ and, JP have a proprietary interest in the PDE4 inhibitor roflumilast.

\section{Authors' information (optional)}

1 Department of Neuropsychology and Psychopharmacology, Faculty of Psychology and Neuroscience, Maastricht University. Limburg Brain Injury Centre, Maastricht University, Maastricht, The Netherlands 2. Department of Neuropsychology and Psychopharmacology, Faculty of Psychology and Neuroscience, Maastricht University, Maastricht, The Netherlands.

3. Department of Psychiatry and Neuropsychology, School of Mental Health and Neuroscience, Faculty of Health, Medicine and Life Sciences, Maastricht University, Maastricht, The Netherlands

4. Department of Neuropsychology and Psychopharmacology, Faculty of Psychology and Neuroscience, Maastricht University. Limburg Brain Injury Centre, Maastricht University, Maastricht, The Netherlands

\section{References}

1. Pollock A, St George B, Fenton M, Firkins L. Top ten research priorities relating to life after stroke. The Lancet Neurology. 2012;11(3):209.

2. Nys G, Van Zandvoort M, De Kort P, Van der Worp H, Jansen B, Algra A, et al. The prognostic value of domain-specific cognitive abilities in acute first-ever stroke. Neurology. 2005;64(5):821-7. 
3. Nys G, Van Zandvoort M, Van Der Worp H, De Haan E, De Kort P, Jansen B, et al. Early cognitive impairment predicts long-term depressive symptoms and quality of life after stroke. Journal of the neurological sciences. 2006;247(2):149-56.

4. Patchick EL, Horne M, Woodward-Nutt K, Vail A, Bowen A. Development of a patient-centred, patient-reported outcome measure (PROM) for post-stroke cognitive rehabilitation: qualitative interviews with stroke survivors to inform design and content. Health Expectations. 2015;18(6):321324.

5. Dougall D, Poole N, Agrawal N. Pharmacotherapy for chronic cognitive impairment in traumatic brain injury. Cochrane Database of Systematic Reviews. 2015(12).

6. van Lieshout EC, van Hooijdonk RF, Dijkhuizen RM, Visser-Meily JA, Nijboer TC. The effect of noninvasive brain stimulation on poststroke cognitive function: a systematic review. Neurorehabilitation and neural repair. 2019;33(5):355-74.

7. Reneerkens OA, Rutten K, Steinbusch HW, Blokland A, Prickaerts J. Selective phosphodiesterase inhibitors: a promising target for cognition enhancement. Psychopharmacology. 2009;202(1):419-43.

8. Kato H, Araki T, Itoyama Y, Kogure K. Rolipram, a cyclic AMP-selective phosphodiesterase inhibitor, reduces neuronal damage following cerebral ischemia in the gerbil. European journal of pharmacology. 1995;272(1):107-10.

9. Schreiber R, Hollands R, Blokland A. A Mechanistic Rationale for PDE-4 Inhibitors to Treat Residual Cognitive Deficits in Acquired Brain Injury. Current neuropharmacology. 2020;18(3):188-201.

10. Soares LM, Prickaerts J, Milani H, Del Bel E, Wilhelm Maria Steinbusch H, Maria Weffort de Oliveira R. Phosphodiesterase inhibition as a therapeutic target for brain ischemia. CNS \& Neurological Disorders-Drug Targets (Formerly Current Drug Targets-CNS \& Neurological Disorders). 2015;14(8):1012-23.

11. Yang F, Sumbria RK, Xue D, Yu C, He D, Liu S, et al. Effects of PDE4 pathway inhibition in rat experimental stroke. Journal of pharmacy \& pharmaceutical sciences: a publication of the Canadian Society for Pharmaceutical Sciences, Societe canadienne des sciences pharmaceutiques. 2014;17(3):362.

12. Wilson NM, Titus DJ, Oliva Jr AA, Furones $C$, Atkins CM. Traumatic brain injury upregulates phosphodiesterase expression in the hippocampus. Frontiers in systems neuroscience. 2016;10:5.

13. Vogel III EW, Morales FN, Meaney DF, Bass CR, Morrison III B. Phosphodiesterase-4 inhibition restored hippocampal long term potentiation after primary blast. Experimental neurology. 2017;293:91-100.

14. Titus DJ, Wilson NM, Freund JE, Carballosa MM, Sikah KE, Furones C, et al. Chronic cognitive dysfunction after traumatic brain injury is improved with a phosphodiesterase 4B inhibitor. Journal of Neuroscience. 2016;36(27):7095-108.

15. Zhang H-T. Cyclic AMP-specific phosphodiesterase-4 as a target for the development of antidepressant drugs. Current pharmaceutical design. 2009;15(14):1688-98.

16. Blokland A, Menniti FS, Prickaerts J. PDE inhibition and cognition enhancement. Expert Opinion on Therapeutic Patents. 2012;22(4):349-54.

17. Prickaerts J, Heckman PR, Blokland A. Investigational phosphodiesterase inhibitors in phase I and phase II clinical trials for Alzheimer's disease. Expert opinion on investigational drugs. 2017;26(9):1033-48.

18. Puhan M. Phosphodiesterase 4 inhibitors for chronic obstructive pulmonary disease. Cochrane Database of Systematic Reviews. 2011(5).

19. Van Duinen M, Sambeth A, Heckman P, Smit S, Tsai M, Lahu G, et al. Acute administration of roflumilast enhances immediate recall of verbal word memory in healthy young adults. Neuropharmacology. 2018;131:31-8.

20. Blokland A, Van Duinen MA, Sambeth A, Heckman PR, Tsai M, Lahu G, et al. Acute treatment with the PDE4 inhibitor roflumilast improves verbal word memory in healthy old individuals: a double-blind placebo-controlled study. Neurobiology of aging. 2019;77:37-43.

21. Gilleen J, Farah Y, Davison C, Kerins S, Valdearenas L, Uz T, et al. An experimental medicine study of the phosphodiesterase-4 inhibitor, roflumilast, on working memory-related brain activity and episodic memory in schizophrenia patients. Psychopharmacology. 2018:1-11.

22. Berry-Kravis EM, Harnett MD, Reines SA, Reese MA, Ethridge LE, Outterson AH, et al. Inhibition of phosphodiesterase-4D in adults with fragile $X$ syndrome: a randomized, placebo-controlled, phase 2 clinical trial. Nature Medicine. 2021;27(5):862-70.

23. Zhang J, Gong Y, Zhao Y, Jiang N, Wang J, Yin X. Post-stroke medication adherence and persistence rates: a meta-analysis of observational studies. Journal of neurology. 2019:1-9 
24. Van Der Elst W, Van Boxtel MP, Van Breukelen GJ, Jolles J. Rey's verbal learning test: normative data for 1855 healthy participants aged 24-81 years and the influence of age, sex, education, and mode of presentation. Journal of the International Neuropsychological Society. 2005;11(3):290-302.

25. Jaeger J. Digit symbol substitution test: the case for sensitivity over specificity in neuropsychological testing. Journal of clinical psychopharmacology. 2018;38(5):513.

26. Smith SR, Servesco AM, Edwards JW, Rahban R, Barazani S, Nowinski LA, et al. Exploring the validity of the comprehensive trail making test. The Clinical Neuropsychologist. 2008;22(3):507-18.

27. Royle J, Lincoln NB. The Everyday Memory Questionnaire-revised: Development of a 13-item scale. Disability and Rehabilitation. 2008;30(2):114-21.

28. Makatura TJ, Lam C, Leahy BJ, Castillo MT, Kalpakjian CZ. Standardized memory tests and the appraisal of everyday memory. Brain Injury. 1999;13(5):355-67.

29. Post MW, van der Zee CH, Hennink J, Schafrat CG, Visser-Meily JM, van Berlekom SB. Validity of the utrecht scale for evaluation of rehabilitation-participation. Disability and rehabilitation. 2012;34(6):478-85.

30. Zigmond AS, Snaith RP. The hospital anxiety and depression scale. Acta psychiatrica scandinavica. 1983;67(6):361-70.

31. Bjelland I, Dahl AA, Haug TT, Neckelmann D. The validity of the Hospital Anxiety and Depression Scale: an updated literature review. Journal of psychosomatic research. 2002;52(2):69-77. 